\title{
The Quantum Triple-Slit Experiment and Dark Energy
}

\author{
Mohamed S. El Naschie \\ Department of Physics, University of Alexandria, Alexandria, Egypt \\ Email: Chaossf@aol.com
}

How to cite this paper: El Naschie, M.S. (2017) The Quantum Triple-Slit Experiment and Dark Energy. Open Journal of Microphysics, 7, 31-35.

https://doi.org/10.4236/ojm.2017.72002

Received: January 5, 2017

Accepted: January 22, 2017

Published: January 25, 2017

Copyright $\odot 2017$ by author and Scientific Research Publishing Inc. This work is licensed under the Creative Commons Attribution International License (CC BY 4.0).

http://creativecommons.org/licenses/by/4.0/

\begin{abstract}
The experimental discovery of looped light in a three-slit experiment is interpreted as a strong justification of the Peano-Hilbert spacetime model. This in turn entails the existence of a dark energy density in full agreement with previous analysis as well as accurate measurements and observations.

\section{Keywords}

Triple-Slit Experiment, Looped Light, Peano-Hilbert Spacetime, Dark Energy, E-Infinity, Cantorian Spacetime, Quantum Double-Slit Experiment, Ord-El Naschie Quantum Spacetime Model
\end{abstract}

\section{Introduction}

A very recent experimental finding [1] [2] [3] apparently reveals for the first time clear cut evidence for a looped travelling path of light [1]. In the present work we argue that this is not a rare event but is what the quantum fine structure of spacetime itself imposes on the movement of light's photons. Consequently the said experiment is interpreted as confirmation of the Cantorian fractal proposal [4] [5] which is based upon the Peano-Hilbert spacetime model presented in the eighties of the last century [6] [7] [8]. It is argued further that once the fractal Peano-Hilbert model of spacetime is accepted as a fact, the physical reality of dark energy and its 95.5 percent density follows suit [9] [10].

\section{The Peano-Hilbert Model of Spacetime and the Findings of the Triple-Slit Experiment}

In several early publications [6] [7] [8] going back to the eighties and nineties of the last century the author utilized a model due to the Canadian-English physicist G. Ord [7] to argue for the Cantorian fractal nature of quantum spacetime [4] [8] [9] [10]. An early example of this effort bares a striking resemblance to the 
recent experimental finding of Ref. [1]. Rather than repeating the mathematical analysis of this paper we think nothing can be simpler and far more convenient for the reader than comparing Figure 1 of Ref. [2] with Figure 2 of our earlier publication, Ref. [8]. However we must stress that the looping of light is not a rare event but rather the very structure of spacetime at the quantum scale. In other words the experiment in Ref. [1] merely used various techniques by which the micro structure was blown up to the more pronounced picture shown in the results of Ref. [1]. In this way we could state with a high degree of confidence that the triple-slit experiment of Ref. [1] has established the fractal structure of spacetime as a physical reality and not only a theoretical possibility.

The author remembers vividly how Nobel Laureate Ilya Prigogine [11] used to say to him that he liked his model but did not know if spacetime is fractal or not. For this reason many experiments were planned and discussed theoretically but were not carried out. Thus the findings of Ref. [1] at long last achieved what we were trying for years to achieve, namely establish the fractal structure of quantum spacetime [12].

\section{The Crucial Point of Topological Mass}

There are many ways to argue that the Kaluza-Klein spacetime dimensionality $D=5$ when extended to a fractal one $D=5+\phi^{3}$ where $\phi=(\sqrt{5}-1) / 2$ represents a total topological mass [13] [14] [15] [16]. It is also very reasoned that while the integer part $D_{1}=5$ represents pure spacetime quantum wave like topological mass, the irrational part $D_{2}=\phi^{3}$ represents the quantum particlelike topological mass [13] [15]. Seen that way it becomes a truly easy task to find that the energy density is made up of two parts. The first is that proportional to $D_{1}=5$ and is given by [15]

$$
\gamma_{1}=(5)\left(\frac{1}{2}\right)(V \rightarrow c=\phi)^{2}
$$

where $c=\phi$ is the topological speed of light [9] [10] [13] [14] [15] [16]. In other words $\gamma_{1}$, is nothing but the dark energy density of pure topological spacetime

$$
\gamma_{1}=\gamma(D)
$$

For $D_{2}=\phi^{3}$ on the other hand one finds in an analogous way that [9] [10] [13] [14] [15] [16]

$$
\gamma_{2}=\left(\phi^{3}\right)\left(\frac{1}{2}\right)(V \rightarrow c=\phi)^{2}=\phi^{5} / 2
$$

Thus $\gamma_{2}$ is nothing but the ordinary energy density of the quantum particle

$$
\gamma_{2}=\gamma(O) \text {. }
$$

Adding $\gamma_{1}$ and $\gamma_{2}$ one finds the maximal density $\gamma_{(\max )}=1$ which is expected on the basis of Einstein's well known maximal energy [13] [14] [15] [16]

$$
E=m c^{2} .
$$




\section{Derivation of $D=5+\phi^{3}$}

The fact that $D=4$ is not sufficient because every point in $D=4$ should also be $D=4$ although being a point means $D=0$ is a subject which we discussed at length and resolved by setting [9] [10]

$$
\begin{aligned}
D= & 4+\phi^{3} \\
& 4+\frac{1}{4+{ }_{4} \ldots \cdots} \\
& =4+\phi^{3} .
\end{aligned}
$$

On the other hand unification of Einstein's gravity with electromagnetism requires that $D=5$ [15]. Consequently we say that [9] [10]

$$
D=4 \rightarrow D=4+1=5
$$

implies that

$$
D=4+\phi^{3} \rightarrow D=4+\phi^{3}+1=5+\phi^{3} .
$$

Here the extra dimension could be regarded as embedding $4+\phi^{3}$ or add one for the volume, similar to what we do in high energy physics when counting particles and matching them to the number of isometries corresponding to say a Lie symmetry group manifold [9] [10].

\section{Discussion}

Even a fleeting look at the results of the recent triple-slit experiment looped path of the light reveals its resemblance to the Peano-Hilbert spacetime model proposed decades ago implicitly by G. Ord [7] and explicitly by the present author [4] [5] [6]. A deeper subsequent look reveals even much more and one realizes that the looped path is far from being exotic. In fact it is generic and all that the experimental set up of the said experiment managed to do is amplify the fractal structure of spacetime to the extent of almost direct observation.

Having established this crucial point we go on to discriminate between two parts that make up our $D=5+\phi^{3}$ spacetime. This is [15]

$$
D=D_{1}+D_{2}=5+\phi^{3} .
$$

Here $D_{1}=5$ is related to dark energy via the Einstein equation $E=m c^{2}$ and is given by [13] [14] [15] [16]

$$
E(1)=\frac{1}{2}\left(D_{1}\right)\left(\phi^{2}\right) m c^{2}=\left(5 \phi^{2} / 2\right) m c^{2}=E(D) .
$$

On the other hand $D_{2}=\phi^{3}$ is related to ordinary energy ad given by [13] [14] [15] [16]

$$
E(2)=\left(\frac{1}{2}\right)\left(D_{2}\right)\left(\phi^{2}\right) m c^{2}=\left(\phi^{5} / 2\right) m c^{2}=E(O) .
$$

Adding $E(1)$ and $E(2)$ one finds the most famous ad recognized equation of physics, namely [13] [14] [15] [16]

$$
E=E_{1}+E_{2}=\left(5 \phi^{2} / 2\right)\left(m c^{2}\right)+\left(\phi^{5} / 2\right)\left(m c^{2}\right)=m c^{2}=E(\text { Einstein }) .
$$




\section{Conclusions}

Recent triple-set experiments revealed beyond any doubt that light may traverse the set up in unexpected looped form. These loops, which are reminiscent of the Peano-Hilbert model of spacetime are interpreted here as being the signature of the very structure of real quantum spacetime brought to the fore. It is found that a quantum spacetime dimension consistent with this picture and Cantorian quantum gravity is a fractal Kaluza-Klein dimension $D=5+\phi^{3}$. In turn the corresponding total energy density of the universe follows from that to be [13] [14] [15] [16]

$$
E=\left(5+\phi^{3}\right)\left(\frac{1}{2}\right)(\phi 2) m c^{2}=m c^{2}
$$

where $\phi$ is the Sigalotti topological speed of light. This Einstein maximal energy can thus be considered to consist of two parts. The first part

$$
E(O)=(5)\left(\phi^{2}\right) / 2 m c^{2}
$$

is clearly the dark energy of the cosmos while the second part

$$
E(D)=\left(\phi^{5} / 2\right) m c^{2}
$$

is the measured ordinary energy density which accounts to only 4.5 percent of the total energy.

It is appropriate in view of the above to conclude this conclusion with some remarkable insights of a scientist with a considerable intuitive mind that was always ahead of his mathematics. I mean of course one of my teachers and mentors who is alas no longer with us, the late Nobel Laureate Ilya Prigogine who wrote round 1987 [11]:

"Probably the most fascinating aspect involved in the transition from dynamics to thermodynamics is the deep change in the structure of spacetime".

It seems that Prigogine's insight also applies to the transition from classical to quantum spacetime.

\section{Acknowledgements}

The author is deeply indebted to Prof. O. Rössler for encouraging him to publish Ref. [8] with Figure 2 of that reference. The author admits that without Prof. Rössler's warm support he would not have had the courage to do that. We thus agree completely with P. Dirac that scientific courage is just as important in science as sound thinking.

\section{References}

[1] Magana-Loaiza, O.S., De Leon, I., Mirhosseini, M., et al. (2016) Exotic Looped Trajectories in the Three-Slit Interference. Arxiv:1610.0858V1[quant-Ph].

[2] Sawant, R., Samuel, J., Sinha, A., Sinha, S. and Sinha, U. (2014) Nonclassical Path in Quantum Interference Experiments. Physical Review Letters, 113, 120406. https://doi.org/10.1103/PhysRevLett.113.120406

[3] Zyga, L. (2017) Physicists Detect Exotic Looped Trajectories of Light in Three-Slit 
Experiment. Physics Org.

https://phys.org/news/2017-01-physicists-exotic-looped-trajectories-three-slit.html

[4] El Naschie, M.S. (2004) A Review of E-Infinity Theory and the Mass Spectrum of High Energy Particle Physics. Chaos, Solitons \& Fractals, 19, 209-236.

https://doi.org/10.1016/S0960-0779(03)00278-9

[5] El Naschie, M.S. (2005) The Two-Slit Experiment as the Foundation of E-Infinity of High Energy Physics. Chaos, Solitons \& Fractals, 25, 509-514. https://doi.org/10.1016/j.chaos.2005.02.016

[6] El Naschie, M.S. (1993) On Turbulence and Complex Dynamics in a Four Dimensional Peano-Hilbert Space. Journal of The Franklin Institute, 330, 183-198. https://doi.org/10.1016/0016-0032(93)90029-T

[7] Ord, G. (1983) Fractal Space-Time: A Geometric Analogue of Relativistic Quantum Mechanics. Journal of Physics A: Mathematical and General, 16, 1869-1884. https://doi.org/10.1088/0305-4470/16/9/012

[8] El Naschie, M.S. (1994) Young Double-Slit Experiment, Heisenberg Uncertainity Principle and Cantorian Spacetime. Chaos, Solitons \& Fractals, 4, 403-409. https://doi.org/10.1016/0960-0779(94)90053-1

[9] Marek-Crnjac, L. and He, J.-H. (2013) An Invitation to El Naschie's Theory of Cantorian Spacetime and Dark Energy. International Journal of Astronomy and Astrophysics, 3, 464-471. https://doi.org/10.4236/ijaa.2013.34053

[10] Helal, M.A., Marek-Crnjac, L. and He, J.-H. (2013) The Three Page Guide to the Most Important Results of M. S. El Naschie's Research in E-Infinity Quantum Physics and Cosmology. Open Journal of Microphysics, 3, 141-145. https://doi.org/10.4236/ojm.2013.34020

[11] Prigogine and Elskens, Y. (1987) Irreversibility, Stochasticity and Non-Locality in Classical Dynamics. In: Hiley, B. and Peat, D., Eds., Quantum Implications, Routledge, London/New York, 205-223.

[12] El Naschie, M.S. (1998) Some Tentative Proposals for the Experimental Verification of Cantorian Micro Spacetime. Chaos, Solitons \& Fractals, 9, 143-144. https://doi.org/10.1016/S0960-0779(97)00175-6

[13] El Naschie, M.S. (2016) Cantorian-Fractal Kinetic Energy and Potential Energy as the Ordinary and Dark Energy Density Respectively. Natural Science, 8, 511-540. https://doi.org/10.4236/ns.2016.812052

[14] El Naschie, M.S. (2017) Quantum Disentanglement as the Physics behind Dark Energy. Open Journal of Microphysics, 7, 1-27. https://doi.org/10.4236/ojm.2017.71001

[15] El Naschie, M.S. (2016) On a Fractal Version of Witten's M-Theory. Journal of Astronomy \& Astrophysics, 6, 135-144. https://doi.org/10.4236/ijaa.2016.62011

[16] El Naschie, M.S. (2016) The Emergence of Spacetime from the Quantum in Three Steps. Advances in Pure Mathematics, 6, 446-454. https://doi.org/10.4236/apm.2016.66032 
Submit or recommend next manuscript to SCIRP and we will provide best service for you:

Accepting pre-submission inquiries through Email, Facebook, LinkedIn, Twitter, etc. A wide selection of journals (inclusive of 9 subjects, more than 200 journals)

Providing 24-hour high-quality service

User-friendly online submission system

Fair and swift peer-review system

Efficient typesetting and proofreading procedure

Display of the result of downloads and visits, as well as the number of cited articles Maximum dissemination of your research work

Submit your manuscript at: http://papersubmission.scirp.org/

Or contact ojm@scirp.org 